INPLASY

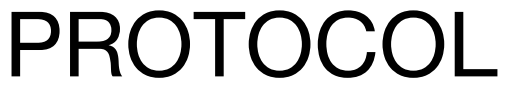

To cite: Gao et al. Exposure to antipsychotics and breast cancer risk: a systematic review and meta-analysis. Inplasy protocol 202220072. doi:

10.37766/inplasy2022.2.0072

Received: 18 February 2022

Published: 19 February 2022

Corresponding author: Zheng Gao

2966526665@qq.com

Author Affiliation:

Department of Second

Hospital of Hebei Medical

University, P. R. China.

Support: Not received yet.

Review Stage at time of this submission: Formal screening of search results against eligibility criteria.

Conflicts of interest:

None declared.

\section{Exposure to antipsychotics and breast cancer risk: a systematic review and meta-analysis}

Gao, Z1; Xi, Y2; Shi, H³ Xu, W4; Ni, J5; Zhang, K6.

Review question / Objective: Whether antipsychotics are associated with the risk of breast cancer in females.

Condition being studied: Breast cancer is the main disease to be studied. The main exposure to be studied is antipsychotics.

Information sources: Online databases were used, including Embase, Scopus, PubMed, Web of Science, Cochrane library, and MEDLINE. The Library of Hebei Medical University was browsed for related books and documents with the administrators' help.

INPLASY registration number: This protocol was registered with the International Platform of Registered Systematic Review and Meta-Analysis Protocols (INPLASY) on 19 February 2022 and was last updated on 19 February 2022 (registration number INPLASY202220072).

\section{INTRODUCTION}

Review question / Objective: Whether antipsychotics are associated with the risk of breast cancer in females.

Condition being studied: Breast cancer is the main disease to be studied. The main exposure to be studied is antipsychotics.

\section{METHODS}

Participant or population: We will include general individuals. Patients with primary hyperprolactinemia not caused by psychotropics at beginning of the follow-up work should be excluded. Cases of metastatic cancer unrelated to psychotropics, patients with multiple immune diseases raised by 
perimenopausal endocrine disorders should also not be used.

Intervention: We will include observational studies, including retrospective cohort studies, case-control studies, and prospective cohort studies. The exposure we will study is mainly antipsychotics.

Comparator: We will include observational studies, including retrospective cohort studies, case-control studies, and prospective cohort studies. The comparators will be those individuals exposed to antipsychotics or not.

Study designs to be included: We will include observational studies, including retrospective cohort studies, case-control studies, and prospective cohort studies.

Eligibility criteria: We will exclude studies that may lead to an incredible conclusion. Retrospective or prospective cohort studies and case-control studies will be accepted. We will exclude randomized controlled trials for the lack of relevant results, as introduced later. And the difference in study design may bring uncertainty to the evidence provided in this study. The study type should be clearly defined. Observational studies should clearly introduce the sources of epidemiological data. Sources such as hospital medical records, public health databases, or insurance agencies will be acceptable. Case-control studies should provide a clear selection criterion of the populations in control groups. And the baseline characteristics of the control groups should be strictly the same as the case groups. Participants in each group should be sufficient to avoid contingency, which means 100 participants at least for each group. The follow-up work should be already completed, ensuring the whole data is available. Criteria of breast cancer or antipsychotics should be defined clearly, which should not be inconsistent with the universal knowledge. Studies that got "Critical risk" overall during quality assessments will be excluded to avoid evidence of bed credibility. Patients with primary hyperprolactinemia not caused by psychotropics at the beginning of the follow-up work should be excluded. Cases of metastatic cancer unrelated to psychotropics, patients with multiple immune diseases raised by perimenopausal endocrine disorders should also not be used. Non-populationbased virtual statistical models will not be accepted. Review articles, case reports, or protocols will not be applied in the present systematic review.

Information sources: Online databases were used, including Embase, Scopus, PubMed, Web of Science, Cochrane library, and MEDLINE. The Library of Hebei Medical University was browsed for related books and documents with the administrators' help.

Main outcome(s): To evaluate the proportion of breast cancer in 1. Individuals exposed to antipsychotics or not. 2. Individuals exposed to typical antipsychotics or atypical antipsychotics.

Quality assessment / Risk of bias analysis: Quality assessment for each study will be operated using the Risk Of Bias In Nonrandomised Studies - of Interventions (ROBINS-I), on $R$ version 4.1.2 software. One reviewer (G.Z.) will evaluate all the studies to be included in seven domains: 1 . Bias due to confounding 2. Bias due to selection of participants 3 . Bias in classification of interventions 4. Bias due to deviations from intended interventions 5 . Bias due to missing data 6 . Bias in measurement of outcomes 7. Bias in selection of the reported results. Each risk will be labelled as "low risk", "Moderate risk", "Serious risk", and "Critical risk". The overall risk will be evaluated manually following the criteria set previously: Studies with two domains of "Moderate risk" or one domain of higher risk will be thought to be at "Moderate risk"; Studies with three or more risks and one or more "Serious risks" of which will be thought to be at "Serious risk". Studies with two or more "Critical risks" will be excluded.

Strategy of data synthesis: The risk ratio (RR) and its $95 \%$ confidence interval will be 
used to evaluate the proportion of breast cancer in different groups. Sub-group analysis and meta-regression analysis will be used to detect possible sources of heterogeneity. A two-tailed $a=0.05$ is set as the statistical significance. $I^{2}$ test will be calculated as measures of heterogeneity, for which we believe $I^{2}$ values of $25 \%, 50 \%$, $75 \%$ to indicate a low, moderate or high heterogeneity. We will take heterogeneity into account during the evaluation of effect.

Subgroup analysis: Important covariates such as continent, country or study design will be used as a classification basis for subgroup analysis. Subgroup analysis is intended to test heterogeneity and factors that may affect results.

Sensitivity analysis: We will perform sensitivity analyses for each outcome to detect potential sources of heterogeneity and to confirm the reliability of the present evidence. Studies will be excluded one by one for the sensitivity analysis.

Country(ies) involved: China.

Keywords: Breast cancer; antipsychotics; risk factor; atypical antipsychotics.

Contributions of each author:

Author 1 - Zheng Gao.

Email: 2966526665@qq.com

Author 2 - Yin Xi.

Email: 1799591554@qq.com

Author 3 - Hekai Shi.

Email: 2534325742@qq.com

Author 4 - Wei Xu.

Email: xdfxvwei@163.com

Author 5 - Jiyuan Ni.

Email: 1825927754@qq.com

Author 6 - Kaili Zhang.

Email: 13833172900@163.com 